\title{
How organisms guide their actions
}

\author{
David N Lee \\ Perception Movement Action Research Consortium \\ School of Philosophy Psychology and Language Sciences \\ The University of Edinburgh
}

Keywords: action, perception, neural, guidance, information, organism

Abstract. A theory is presented about the information available for guiding purposeful actions by any organism, whether animal or plant, and about how the information is used in guiding actions.

\begin{abstract}
"It is interesting to contemplate an entangled bank, clothed with many plants of many kinds, with birds singing on the bushes, with various insects flitting about, and with worms crawling through the damp earth, and to reflect that these elaborately constructed forms, so different from each other, and dependent on each other in so complex a manner, have all been produced by laws acting around us.” C. Darwin 1859.
\end{abstract}

\begin{abstract}
"Henceforth space by itself, and time by itself, are doomed to fade away into mere shadows, and only a kind of union of the two will preserve an independent reality." N. Minkowski 1908.
\end{abstract}

\section{The nature of organismic action}

How do organisms make the purposeful actions that are the sine qua non of life. Wonders of living action abound - bacteria foraging, leaves orienting to light, bees flitting from flower to flower, bats catching insects on the wing, birds landing delicately on twigs, seabirds plunge-diving after fish, horses leaping fences, cricketers catching balls, babies communicating by crying and cooing, adults in conversation, sopranos singing, dancers leaping, fiddlers fiddling, etc. Also, within bodies there are many wonders of living action: for example, hearts pumping blood, lungs inhaling and exhaling air, muscles contracting and extending, immune cells attacking invaders, kidneys filtering blood. And underpinning all these actions are the actions of trillions of cells.

To support living action anatomical tools have evolved - hearts, hands, feet, claws, beaks, cilia, flagella and so on. However, this is only half the story. Anatomical tools must have evolved handin-glove with intrinsic action-control processes in order for the tools to be used. In this paper we 
seek to understand some of the intrinsic control laws governing living actions. These laws are different from terrestrial physical laws, which describe the motion of inanimate matter, such as Newton's laws of motion. However, the intrinsic control laws of action must harmonize with terrestrial physical laws, because an organism's actions have to fit with, and indeed exploit, terrestrial physical laws. Thus the intrinsic control laws should, in principle, be expressible mathematically so that they marry with mathematical physical laws.

\section{Basic principles of control of organismic action}

(1) Purposeful actions must be prospectively controlled, i.e., controlled ahead in time. For example, drivers have to prospectively control their braking when stopping. Birds have to prospectively flare their wings when landing. Animals (and plants) have to prospectively control their growth. The basic reason why prospective control is necessary is inertia: actions take time to execute and therefore need to be set up in advance of their execution. Prospective control is achieved through an organism's intrinsic control system. This comprises flows of information in the form of patterns of electrochemical power. In animals and plant the intrinsic control system consists of an electrochemical neural system coupled with a chemical endocrine system.

(2) The intrinsic control system has three basic functions:

- Perceptually setting-up and monitoring purposeful actions, to fit in with the external spatiotemporal events in which the organism is immersed.

- Prescribing purposeful actions, to create the intended patterns of flow of action.

- Performing purposeful actions, by regulating the power to the muscles.

(3) Actions of body parts must be coordinated to achieve whole-body action. For example, runners must coordinate actions of the head, trunk, arms, hands, legs and feet to negotiate terrain. A growing tree must coordinate the growth of its branches and leaves to maintain balance and secure its energy needs.

(4) The intrinsic information for action control must be robust. For example, the normal generation and degeneration of neurons must minimally disrupt action control.

\section{Rho/tau theory of intrinsic control of action}

Rho/tau theory is a development of General Tau Theory (Lee et al. 2009). The principal tenets of rho/tau theory are as follows.

\subsection{Action gaps}


Purposeful actions, whether outside or inside an organism's body, entail controlling the closings and openings of action-gaps. Action-gaps can be across a variety of physical dimensions - distance, angle, pressure, and so on. Examples of action-gaps are: the distance gaps between an animal's foot and ground when running, and between a plant's bud and flower when blooming; the angular gaps between initial and final direction when an animal is shifting gaze and when a plant is orienting its leaves to light; the angular gaps between the body and gravity when an animal or plant is maintaining balance; the pressure gaps when an animal is gripping an object and when a root is squeezing through soil.

\subsection{Perceptual information}

Perceptual information about spatio-temporal events external to a body's nervous or endocrine systems, either within or outside the body, is necessary for setting up and monitoring purposeful actions within or outside the body. The information is embodied in the pattern of stimulus-power incident on sensory receptors. Stimulus-power is the power emanating or reflected from a source that is remote from the receptors. It comes in several forms. It is optical (electromagnetic) for seeing, mechanical for touching, acoustic for hearing and echolocating, chemical for smelling and tasting, electrical for electro-sensing, magnetic for magneto-sensing, thermal for heat-sensing, mechanical for sensing gravity, friction and other external forces. Following the inverse-square law of physics, stimulus-power equals the power of the source divided by the square of the distance, $r$, to the source (ignoring disturbances to power transmission, caused e.g., by mist and gusts of wind). That is

$$
P_{\text {stimulus }}=P_{\text {source }} / r^{2}
$$

Thus stimulus-power varies conjointly with source-power and source-distance. Consequently, stimulus-power does not contain information about the distance or power of the source. However, if the source-power is constant, if only for brief periods, then it follows immediately from Eq. (1) that

$\rho\left(P_{\text {stimulus }}\right)=-2 \rho(r)$

where, e.g., $\rho(r)$ equals the relative-rate-of-change of $r$, which equals $\dot{r} / r$. Thus, $\rho(r)$ is directly perceptually specified, whereas $r$ or any of its time derivatives (velocity, acceleration, jerk, etc) are 
not directly perceptually specified (though $r$ can be indirectly perceptually specified in terms of two or more $\rho(r)$ s that provide scaling information: Lee 1980).

\subsection{Stimulus-power to sensory neural-power}

The basic stimulus information, $\rho\left(P_{\text {stimulus }}\right)$, has to be transduced at the sensory receptors into corresponding intrinsic information for use by the organism's neural (or ionic) system. When $P_{\text {stimulus }}$ lies within the sensitivity range of the sensory receptors, it has to be transduced by them into some function of neural-power, $P_{\text {neural }}$, which equals the rate of flow of electrochemical energy in the nervous, or ionic, system). Assuming an unknown amplification or minification of $P_{\text {stimulus }}$ in the transduction process, then

$P_{\text {neural } / \text { sensory }}=a P_{\text {stimulus }}$

where $a$ is the unknown amplification or minification factor. Thus, there is not a 1-1 relation between stimulus power and neural power. Therefore, the neural power cannot specify the stimulus power, or any time derivative of it. However, from Eq. (3) it is easily shown that

$\rho\left(P_{\text {neural } / \text { sensory }}\right)=\rho\left(P_{\text {stimulus }}\right)$

\subsection{Stimulus-power to nocicptive neural-power}

When stimulus-power lies above the sensitivity range of the sensory receptors, and so could cause harm to an animal, it is transduced into nociceptive neural-power by the animal's nociceptors (pain receptors). Again, an unknown proportion of the nociceptive stimulus-power will inevitably be lost or gained in the transduction process. Therefore, the nociceptive neural-power cannot specify the stimulus-power. However, the rho of the nociceptive neural-power will always be equal to the rho of the nociceptive stimulus-power. That is

$$
\rho\left(P_{\text {neural/nociceptive }}\right)=\rho\left(P_{\text {stimulus/nociceptive }}\right)
$$

\subsection{Sensory and nociceptive neural-power}

In animals, sensory and nociceptive neural-power flows from sensory receptors and nociceptors along nerves and across synapses in the periphery, where they are recorded as graded potentials. 
More centrally, to speed up and strengthen transmission, the sensory and nociceptive neuralpowers are rendered discontinuous, by the nervous system amplifying the neural-power by injecting uniform packets of ionic energy at the nodes of Ranvier along axons. These injections effectively give the neural-power a series of boosts. The resulting neural-power packets are recorded as approximately uniform action potentials, of around $100 \mathrm{mV}$ amplitude and duration $1 \mathrm{~ms}$. The rate of flow of action potentials, which is recorded as spike-rate, equals the rate of flow of neural-power. The overall level of neural-power is increased or decreased by this continuous-to-discontinuous conversion process. However, $\rho\left(P_{\text {neural }}\right)$ is independent of the overall level of neural-power, and so remains invariant. Thus the changed neural-power continues to convey the same information in $\rho\left(P_{\text {neural }}\right)$.

\subsection{Prescriptive neural-power}

It is assumed that the rho of prescriptive neural power $\left(\rho\left(P_{\text {neural/prescriptive }}\right)\right.$ is created in the nervous system to determine how the rho of an action-gap should change. Measures of skillful closure of action-gaps by humans, animals and cells (Lee et al. 2009) suggests that one commonly used prescriptive neural-power is

$$
\lambda \rho_{G}=2 \lambda t /\left(t^{2}-T^{2}\right)
$$

where time $t$ is zero at the start of the action-gap, $T$ is the duration of the action-gap and $\lambda$ is a dimensionless velocity parameter that regulates the velocity profile of the movement, and hence the kinematics of the movement.

\subsection{Regulating momentum}

A purposeful movement needs to be directed not only to the right place at the right time but also with the right momentum. For example, when a seabird is spearing fish the momentum must be forceful, but when feeding the fish to its young the momentum must be gentle. Neurally regulating the velocity parameter, $\lambda$, and the duration parameter, $T$ (Eq.6) are two ways of regulating the momentum of a rhoG-guided movement. The third way is regulating the mass that is being moved, but we here assume the mass is constant. Decreasing the $T$ of rhoG-guided movement of a given magnitude will obviously increase its momentum. As regards neural regulation of momentum by the velocity parameter, $\lambda$, a rhoG-guided movement first accelerates at a non-constant rate and then decelerates at a non-constant rate, or continues accelerating, depending on the value of the 
dimensionless parameter, $\lambda$. If $\lambda \geq 2$, both velocity and deceleration approach zero at the end of the action gap and so closure is gentle. If $1<\lambda<2$, deceleration theoretically approaches infinity at the end of the action gap, thus closure is forceful. If $\lambda \leq 1$ the movement continues to accelerate and so again closure is forceful. The effect of $\lambda$ on momentum is illustrated in Fig. 1. Thus, a seabird must use $\lambda<2$ when spearing fish, and $\lambda \geq 2$ when feeding the fish to its young.

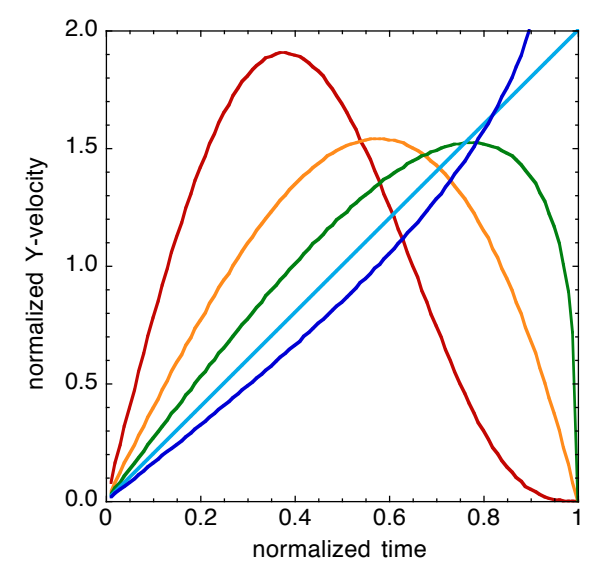

Fig. 1. Effect of the velocity parameter, $\lambda$, on the rhoG-guidance of the velocity of closure of an action gap, $Y$, following the equation $\rho(Y)=\lambda \rho_{G}(T)$ (Eq.6). Red, $\lambda=4$. Orange, $\lambda=2$. Green, $\lambda=4 / 3$. Light blue, $\lambda=1$. Blue, $\lambda=4 / 5 . Y$ and movement duration, $T$, are each normalized to unity for illustration.

\subsection{Experimental evidence for rhoG-guidance}

This includes human neonates regulating suction when suckling (Craig \& Lee 1999), gesturing with the arms (Delafield-Butt et al. 2018) and vocalizing (Lee et al. 2017); adults raising food to the mouth (Lee et al. 1999), adults shifting gaze (Lee et al. 2009), golfers putting (Craig et al., 2000), pilots flying (Padfield et al, 2003)), musicians singing and playing (Schogler et al. 2008, Lee \& Schogler 2009); hummingbirds docking on a feeder (Delafield-Butt et al. 2010). There is also neural-cum-behavioural evidence in the rhoG-guidance of electrical activity in the basal ganglia that correlates with the rhoG-guidance of the hand of monkeys reaching to targets (Lee et al., 2018).

\subsection{Motor neural-power}

The rho of prescriptive neural-power, $\rho\left(P_{\text {neural/prescriptive }}\right)$, specifies how the rho of an action gap should change. The rhos of sensory neural-power, $\rho\left(P_{\text {neural/sensory }}\right)$, and of nociceptive neural-power, $\rho\left(P_{\text {neural/nociceptive }}\right)$ specify how the rho of the action-gap is actually changing. These two rhos must be 
combined in the nervous system to create a rho of motor neural-power, $\rho\left(P_{\text {neural } / \text { motor }}\right)$, to drive the muscles. How this is achieved is suggested by the experiment mentioned earlier, where rhesus monkeys controlled a manipulandum to move a cursor across an action-gap to goal along a linear track (Lee et al. 2018). The action-gap was recorded simultaneously with the electrical spike-rate (neural-power) in four basal ganglia: GPe, GPi, STN and ZI. The results indicated that (i) the action-gap was rhoG-guided; (ii) GPe was implicated in creating $\rho\left(P_{\text {neural/prescriptive }}\right)$, prescribing the action-gap; (iii) STN was implicated in registering $\rho\left(P_{\text {neural/sensory }}\right)$, monitoring the action-gap; and (iv) $\rho\left(P_{\text {neural/prescriptive }}\right)$ and $\rho\left(P_{\text {neural/sensory }}\right)$ were linearly combined in ZI to produce $\rho\left(P_{\text {neural/motor }}\right)$ to guide the muscles.

\subsection{Coordinating motor-elements}

Purposeful actions generally involve coordinating many joints and muscles. How this coordination is achieved has long been a puzzle. A solution to the puzzle could have practical applications in rehabilitation therapy, sports training, robotic design, and many other areas.

The coordination of the many and varied arrangements of muscles that move bodily joints would appear to be very complicated. However, all animals great and small appear to achieve such coordination with ease. This suggests there might be a simple universal principle involved.

To try to discover this principle we start by considering the motor-elements involved in flexing or extending a joint. A motor-element is conceived as the straight compressible/extensible connection between the ends of two bones that are flexibly connected at their other ends at a joint (e.g., a knee). The motor-element is controlled by a set of muscles connected to the bones. A motor-element may rotate in 3-D - for example, the motor-element between the shoulder and wrist, with intervening joint at the elbow. Thus, in general, a motor-element may be represented as a vector, and limbs and vertebrae as 3-D chains of motor-element vectors. A nervous system needs to program and perceptually monitor the rhos of the lengths and directions of these motor-element vectors in order to regulate the rho of the gap between the effector (e.g. a hand) at the end of the chain of motor-elements and a goal object, as when catching a moving ball. How might a nervous system solve this coordination problem, using only fundamental perceptual information? Let us break the problem down into a series of component problems, as follows.

\subsection{RhoG-guidance of an effector by a single motor-element}

First note that a nervous, or ionic, system has to regulate the lengths and directions of pull of the muscles controlling a joint, rather than the forces applied by the muscles. This is because muscular 
forces do not specify joint movement, because unpredictable external forces act on joints as well (Bernstein 1967).

Consider a motor element, length $m$, connecting a point $P$ on the body to an effector $E$ via a joint $J$. Suppose $E$ has to be moved across a gap, length $x$, to a destination $D$, a distance $d$ from $P$. $D$ may or may not be moving with respect to $P$. Thus, in general, $m, x$ and $d$ are all functions of time. How must $\rho_{m}$ be regulated so that $\rho_{x}$ equals a prescribed rho, $\rho_{\text {prescribed }}$ ? The solution is straightforward ${ }^{1}: \rho_{m}$ needs to be constantly regulated such that

$\rho_{m}=\left[\rho_{d}-(x / d) \rho_{\text {prescribed }}\right] /[1-(x / d)]$

where $\rho_{d}$ and $x / d$ are directly perceptible and $\rho_{\text {prescribed }}$ is specified by the organism. Thus $\rho_{m}$ is specified by sensory and prescriptive rho information.

\subsection{Chaining motor-elements}

Now consider replacing the single motor-element, $m$, with a chain of vector motor-elements, $\left\{m_{i}\right\}$, where $i$ indexes the motor-element. We hence have an abstract model of an animal's musculoskeletal system moving an effector in a straight line to a destination. The question is: how should the lengths of the individual motor-elements be regulated so that $\rho_{m}=\rho_{\text {prescribed }}$ ? In principle, there are an infinite number of ways of doing this. However, a parsimonious and energyefficient solution would be to make the $\rho$ vector, $\rho_{m_{i}}$, of each motor-element, $i$, equal to $\rho_{\text {prescribed }}$, the prescribed rho of the action-gap between the effector and the destination. This would mean that the relative rate of change of lengths of motor elements in the 1-D chain would be uniform and so the chain would stretch or contract uniformly. On the other hand, if one or more of the motorelements in the chain had a different rho than the rest, this would disrupt the uniformity of the action, introduce local changes in tension, and make the action less energy- efficient. Such could occur, for example, if a muscle were injured or if the interface with the environment (e.g., shoe, glove, or prosthesis) had different characteristics to the rest of the chain.

\footnotetext{
${ }^{1}$ If $m=x-d$ then $\rho_{m}=(\dot{d}-\dot{x}) /(d-x)=\left(d \rho_{d}-x \rho_{x}\right) /(d-x)$. Rearranging this equation and putting $\rho_{x}=\rho_{\text {prescribed }}$ we have $\rho_{m}=\left[\rho_{d}-(x / d) \rho_{\text {prescribed }}\right] /[1-(x / d)]$
} 


\section{References}

Bernstein, N. (1967). The co-ordination and regulation of movements. Oxford, Pergamon.

Craig, C. M. \& Lee, D. N. (1999). Neonatal control of nutritive sucking pressure: evidence for an intrinsic $\tau$-guide. Experimental Brain Research, 124, 371-382.

Craig, C. M., Delay, D., Grealy, M. A. \& Lee, D. N. (2000) Guiding the swing in golf putting. Nature, 405, 295-296.

Delafield-Butt, J. T., Galler, A., Schögler, B. \& Lee, D. N. (2010). A perception-action strategy for hummingbirds. Perception, 39, 1172-1174.

Delafield-Butt, J. T., Freer, Y., Perkins, J., Skulina, D., Schögler, B. \& Lee, D.N. (2018). Prospective Organisation of Neonatal Arm Movements: A motor foundation of embodied agency, disrupted in premature birth. Developmental Science (in press).

Lee, D. N. (1980). The optic flow-field: the foundation of vision. Philosophical Transactions of the Royal Society London B, 290:, 169-179.

Lee, D. N., Craig, C. M. \& Grealy, M. A. (1999). Sensory and intrinsic coordination of movement. Proceedings of the Royal Society of London B, 266, 2029-2035.

Lee, D. N., Bootsma, R. J., Frost, B. J., Land, M. \& Regan, D. (2009). General Tau Theory: evolution to date. Special Issue: Landmarks in Perception. Perception, 38, 837-858.

Lee, D. N., \& Schogler, B. (2009). Tau in musical expression. In S. Malloch \& C. Trevarthen (Eds.), Communicative Musicality: Exploring the basis of human companionship. Oxford: Oxford U. P.

Lee, D.N., Obregon, M. \& Delafield-Butt, J. (2017) TauG-guidance of neonatal vocalizing. doi: https://doi.org/10.1101/143156.

Lee, D.N., Georgopoulos, A.P. \& Pepping, G-J. (2018) Prospective control of movement in the basal ganglia. doi: https://doi.org/10.1101/256347.

Padfield, G. D., Lee, D. \& Bradley, R. (2003). How do pilots know when to stop, turn or pull up? Developing guidelines for vision aids. J. American Helicopter Society, 48, 108-119.

Schogler, B., Pepping, G-J. \& Lee, D. N. (2008). TauG-guidance of transients in expressive musical performance. Experimental Brain Research, 198, 361-372. 